\title{
The neutrophil lymphocyte ratio is associated with breast cancer prognosis: an updated systematic review and meta-analysis
}

This article was published in the following Dove Press journal:

OncoTargets and Therapy

8 September 2016

Number of times this article has been viewed

\author{
Bajin Wei ${ }^{1,2}$ \\ Minya Yao' \\ Chunyang Xing ${ }^{2}$ \\ Wei Wang ${ }^{2}$ \\ Jia Yao' \\ Yun Hong' \\ Yu Liu' \\ Peifen Fu ${ }^{1,2}$ \\ 'Diagnosis and Treatment of Breast \\ Diseases Center, ${ }^{2}$ Key Laboratory \\ of Organ Transplantation, Key \\ Laboratory of Combined Multi-Organ \\ Transplantation, The First Affiliated \\ Hospital, School of Medicine, Zhejiang \\ University, Hangzhou, People's \\ Republic of China
}

\begin{abstract}
Breast cancer (BC) is the most common female malignancy within the spectrum of human cancer. One promising way to reduce the mortality and morbidity of $\mathrm{BC}$ is to explore novel diagnostic markers for early diagnosis and prognostication. The neutrophil lymphocyte ratio (NLR) is a good reflection of inflammation, which plays an important role in tumor progression and metastasis. However, the association between NLR and BC prognosis remains unclear. The aim of this meta-analysis is to explore the prognostic value of NLR in BC. Among the screened references in the database, 12 eligible studies were identified in this study. Patients with a higher NLR had a shorter disease-free survival (hazard ratio $=1.46,95 \%$ confidence interval: $1.12-1.90, P=0.044$ ) and overall survival (hazard ratio $=2.03,95 \%$ confidence interval: $1.41-2.93, P<0.001)$. In the subgroup analysis of NLR and disease-free survival, the studies from Eastern countries had a positive result with perfect homogeneity $\left(I^{2}=0\right)$; however, this homogeneity has not been achieved in studies from Western countries. In the subgroup analysis of the NLR and overall survival, the results of the univariate and multivariate analyses were completely different, with different heterogeneity. In the luminal A and luminal B subtypes, we found that there was no association between the NLR and overall survival in the BC patients. Positive results were obtained in the analyses of the human epidermal growth factor receptor 2 (HER2)-positive and triple-negative BC subtypes. In conclusion, this meta-analysis suggests that NLR is a good prognostic marker for BC, and patients with a higher NLR have poorer prognoses. Future studies should perform more detailed investigations to decrease heterogeneity and determine the appropriate cut-off values for different races.
\end{abstract}

Keywords: disease-free survival, overall survival, inflammatory factor, lymphocytosis, lymphocytopenia, neutrophilic leukocytosis, neutrophilicgranulocytopenia, prognosis

\section{Background}

Although the diagnosis and treatment of breast cancer (BC) has improved in the past few years, it is still the most common female malignancy worldwide. ${ }^{1}$ In 2012, 1.7 million women were diagnosed with BC, and >521,000 women died of the disease. ${ }^{2}$ A very steep increase in incidence and mortality was observed in 2012 compared to 2008 (21.2\% and 13.6\%, respectively). Developing countries have a lower incidence of $\mathrm{BC}$ than developed countries, but the mortality is high due to the lack of diagnosis and treatment. ${ }^{3}$ Due to its complex nature, the progression and prognosis of BC are not yet well understood. Some of the proven molecular prognostic assays, such as Oncotype $\mathrm{dx}^{\circledR}$ and Pam-50 ror $^{\circledR}$, are expensive and inaccessible to most patients. ${ }^{4}$ Therefore, predictive factors with economical and practical advantages are desirable.
Correspondence: Peifen Fu

Diagnosis and Treatment of Breast

Diseases Center, The First Affiliated

Hospital, School of Medicine, Zhejiang

University, 79 Qingchun Road, Hangzhou,

Zhejiang, 310003 , People's Republic of

China

Tel $+8657 \mid 87236852$

Fax+86 57I8723 5I25

Email fupeifen@hotmail.com 
A routine blood test is widely used as a traditional detection method prior to surgery. Previous studies found that the neutrophil lymphocyte ratio (NLR) could provide a prompt representation of the state of inflammation, which might play key roles in tumor growth, progression, invasion, and metastasis. ${ }^{5,6}$ Many inflammatory factors are associated with $\mathrm{BC}$ prognosis. The preoperative C-reactive protein level is an independent prognostic factor of disease-free survival (DFS) and overall survival (OS) in node-negative BC. ${ }^{7}$ Human epidermal growth factor receptor 2 (HER2)-negative patients with increased interleukin (IL)-6 and -8 levels have a shorter relapse-free survival. ${ }^{8}$ The coexpression of IL- 6 and tumor necrosis factor- $\alpha$ is a better predictive marker of BC survival than either factor in isolation. ${ }^{9}$ However, the association between the preoperative NLR and prognostic value in BC patients remains controversial. It should be noted that some studies indicate patients with a lower NLR had a better prognosis, whereas other studies have failed to show an association. ${ }^{10-22}$ Thus, a meta-analysis of the association between the pretreatment NLR and the prognosis of $\mathrm{BC}$ patients is warranted.

\section{Materials and methods}

\section{Search strategy}

A systematic search of the relevant literature was performed in the PubMed and Web of Science databases, including the Science Citation Index Expanded. The deadline for the date of publication was November 30, 2015. Reference lists of the identified studies were also examined to identify additional eligible studies. If new relevant articles were found, an adjusted search was performed. The final search terms were: (neutrophil lymphocyte ratio) OR (lymphocytosis) OR (lymphocytopenia) OR (neutrophilic leukocytosis) OR (neutrophilicgranulocytopenia) OR (NLR) AND (breast tumor OR breast cancer OR breast carcinoma).

\section{Inclusion and exclusion criteria and the literature screening process}

The inclusion and exclusion criteria were developed compared to those used in previous studies. The inclusion criteria were: 1) the articles to be published in English, 2) sufficient data (the hazard ratio [HR] and 95\% confidence interval [CI]) to be provided on the pretreatment NLR and prognosis (DFS or OS), 3) the HR and $95 \%$ CI to be obtained by univariate or multivariate analyses, and 4) the studies to be approved by the local ethics committees. The exclusion criteria were: 1) the studies did not supply the details of the HR and 95\% CI and 2) review articles, nonclinical studies, letters, expert opinions, conference summaries and case reports. The following process was used to screen and evaluate the literature: 1) the search results were combined; 2) duplicate articles were removed; 3) articles with insufficient information were removed; 4) studies that did not meet the language requirement, publication type, or relevant search terms were excluded; and 5) studies of NLR and $\mathrm{BC}$ prognosis were retrieved.

\section{Data extraction}

Two authors (BW and MY) independently reviewed the studies and extracted data from each study. When a disagreement occurred, the article was discussed by all the authors. If the patients were enrolled in more than two studies, only the most recent study was included. The quality was assessed using the Newcastle-Ottawa Quality Assessment Scale, and studies with scores $\geq 6$ were defined as high-quality studies. For each study, the following characteristics were collected: PMID (PubMed Unique Identifier), author's name, region, year of publication, journal title, predominant treatment (surgical or nonsurgical), the number of patients, the HRs, and the $95 \%$ CIs.

\section{Statistical analysis}

All of the data from the enrolled studies were stored and analyzed using STATA SE 12.0 (StataCorp LP, College Station, TX, USA). To analyze the differences according to race, the definition of high and low NLRs (cut-off value) was consistent with the original study. The author's favorite value (the value used in the subsequent multivariate analysis) was chosen if more than two values were reported in the study. The HRs and 95\% CIs from the univariate and multivariate analyses were extracted from each study and used to generate a pooled HR. The detailed association between NLR and OS in estrogen receptor (ER)-positive and/or progesterone receptor (PR)-positive/HER2-negative disease, ER-positive and/or PR-positive/HER2-positive disease, ER-negative/ PR-negative/HER2-positive disease, and ER-negative/PRnegative/HER2-negative disease was also obtained, where possible. Predetermined subgroup analysis was performed according to region (Eastern or Western), year published (up to 2016), the cut-off value ( $<3$ or $\geq 3$, the median value), the patient composition (mixed or not), and the analysis method (univariate or multivariate analysis). Cochran's Q test and the Higgins $I^{2}$ statistic were used to assess heterogeneity, and Begg's funnel plot and Egger's linear regression were used to evaluate publication bias. The result was defined as heterogeneous when the Higgins $I^{2}$ statistic was $\geq 50 \%$ or the $P$-value was $\leq 0.10$ for the $\mathrm{Q}$ test. The random-effects model was used if there was heterogeneity among studies; otherwise, the fixed-effects model was used. The HR and 95\% CI were considered statistically significant when $P<0.05$ and the $95 \%$ CIs did not overlap in all statistical analyses. Moreover, both 
an $\mathrm{HR}$ and a $95 \% \mathrm{CI}$ of $>1$ revealed that the BC patients with high NLRs had poor prognoses (a short DFS or OS). A positive result reflects that the NLR is associated with the prognosis of $\mathrm{BC}$ patients, whereas a negative result has the opposite meaning.

\section{Results}

\section{Literature searches and study characteristics}

The search, selection, and evaluation strategy, as detailed earlier, identified 12 studies appropriate for inclusion in this meta-analysis. A flowchart of the literature search, selection, and evaluation is shown in Figure 1.

The 12 selected studies from eight different countries included 7,951 patients who were diagnosed with BC. Among them, ten studies presented data on the pretreatment NLR and OS, and eight studies focused on the relationship between the pretreatment NLR and DFS in BC patients. The detailed characteristics of the studies are supplied in Table 1.

\section{NLR is closely associated with BC prognosis}

When considering the overall impact of the NLR on survival in BC patients, we found that the patients with elevated NLRs had shorter DFS (HR $=1.46,95 \%$ CI: $1.12-1.90, P=0.044$, $\left.I^{2}=51.5 \%\right)$ and $\mathrm{OS}(\mathrm{HR}=2.03,95 \% \mathrm{CI}: 1.41-2.93, P<0.001$, $I^{2}=72.7 \%$ ) (Figure 2).

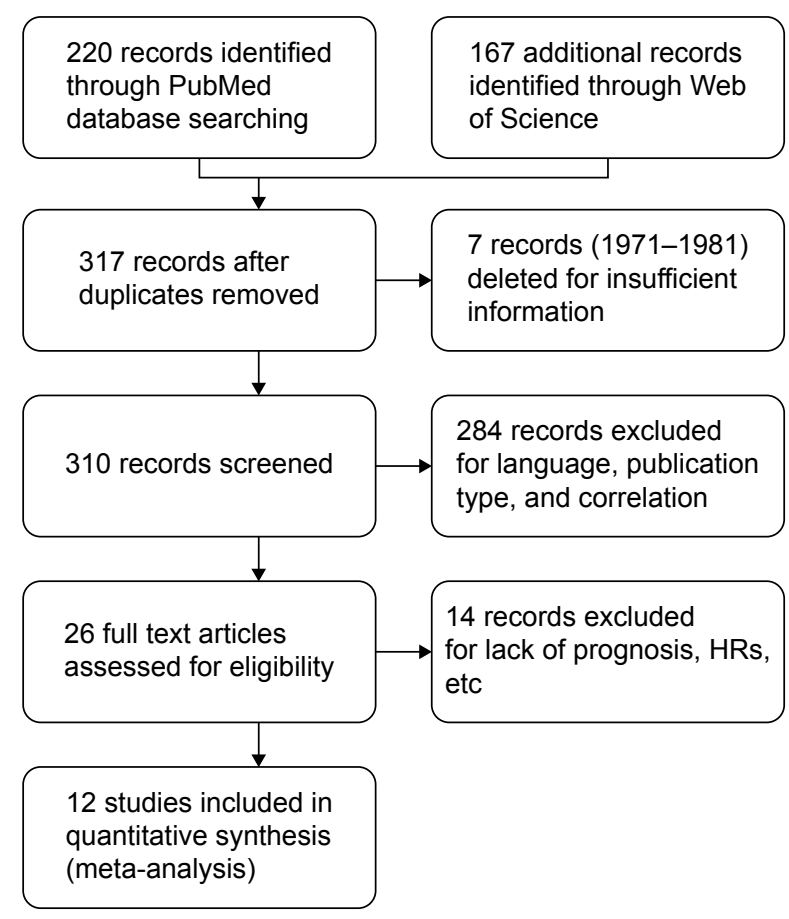

Figure I A flowchart of the literature search and study selection. Abbreviation: HRs, hazard ratios.

\section{Sensitivity analysis}

Each study was successively deleted to assess the influence of individual studies on the pooled HR (Figure 3). The results of the sensitivity analyses revealed that the corresponding pooled HRs were not radically changed.

\section{Subgroup analysis of the NLR and DFS in $B C$ patients}

We found that studies from Eastern countries had positive results, with perfect homogeneity $\left(R^{2}=0\right)$; this homogeneity was not achieved in studies from Western countries (Table 2). The studies in 2015 and 2016 did not change the result of the main analysis, whereas the studies from 2013 and 2014 had negative results, with high heterogeneity. The results remained positive when the cut-off value was $<3$. Only one study included patients with metastasis and the other studies still revealed a positive result, similar to the main analysis. The results of the univariate and multivariate analyses were extremely different, but both analyses revealed slight heterogeneity $\left(I^{2}=0\right)$.

\section{Subgroup analysis of the NLR and OS in $B C$ patients}

Analyses within the same subgroup were performed for NLR and OS in BC patients (Table 3). We found that studies from both Eastern and Western countries had pooled positive results, with high heterogeneity $\left(I^{2}=74.9 \%\right.$, 73.7\%). ${ }^{10-14,16-19,21,22}$ The studies before 2015 presented results similar to the main analysis of NLR and OS in BC patients. ${ }^{13-16,18,19}$ A positive result, with less heterogeneity $\left(I^{2}=36.1 \%\right)$, was obtained from the studies in 2015 and 2016. ${ }^{10,12,17,21,22}$ Three studies included patients with metastasis, and the other studies agreed with the historical findings in the main analysis. ${ }^{10,16,21}$ The results of univariate and multivariate analyses were completely different, with different heterogeneity $\left(I^{2}=0 \%, 71.2 \%\right)$.

\section{NLR and $O S$ in $B C$ subtype patients}

In the luminal A and luminal B subtypes, we found that there was no association between the NLR and OS in the BC patients (Table 4). Positive results were obtained in the analyses of the HER2+ and triple-negative breast cancer subtypes.

\section{Heterogeneity analysis}

We further explored potential mechanisms of heterogeneity in a meta-regression analysis. Five features (region, year published, cut-off value, patient composition, and analysis method) were assessed in both the DFS and OS studies. The analysis method was nearly considered the source of heterogeneity in the analysis of the association between NLR 
Table I The characteristics of the enrolled studies

\begin{tabular}{|c|c|c|c|c|c|c|c|c|c|c|c|}
\hline PIMD & Author & Year & $\begin{array}{l}\text { Countryl } \\
\text { region }\end{array}$ & NOS & Time & Age & Number & $\begin{array}{l}\text { Follow-up } \\
\text { (months) }\end{array}$ & Cut-off & Metastasis & Analysis \\
\hline 26022929 & Koh et al ${ }^{10}$ & 2015 & Malaysia & 7 & 2000-2008 & 52 & $\mathrm{I}, 435$ & ND & $4^{a}$ & $Y_{e s}^{b}$ & OS \\
\hline 26580962 & Jia et $\mathrm{al}^{12}$ & 2015 & PRC & 7 & 2000-2010 & $23-91$ & $\mathrm{I}, 570$ & 79 & 2 & $\mathrm{No}^{c}$ & DFS + OS \\
\hline 25328407 & Yao et $\mathrm{al}^{13}$ & 2014 & PRC & 7 & 2009-201I & $52.4 \pm 10.8$ & 608 & 42 & 2.57 & No & OS \\
\hline 24986572 & Koh et $\mathrm{al}^{14}$ & 2014 & Korea & 8 & $2002-2010$ & 44 (24-7I) & 157 & 21 & 2.25 & No & OS \\
\hline 24982408 & Nakano et al ${ }^{15}$ & 2014 & Japan & 6 & $200 I-201 I$ & $58.9 \pm 11.1$ & 652 & 85.8 & 2.5 & No & DFS \\
\hline 23283648 & Azab et $a^{16}$ & 2013 & America & 6 & 2004-2006 & $63.6 \pm 0.7$ & 437 & 60 & 3.33 & Yes & OS \\
\hline 25884918 & Pistelli et al ${ }^{17}$ & 2015 & Italy & 8 & $2006-2012$ & 53 & 90 & 53.8 & 3 & No & DFS + OS \\
\hline 24935375 & Cihan et $\mathrm{a}^{18}$ & 2014 & Turkey & 8 & 2005-2010 & $55.3 \pm 0.3$ & 350 & ND & 3 & No & DFS + OS \\
\hline 244646 II & Forget et al ${ }^{19}$ & 2014 & Belgium & 8 & 2003-2008 & $27-89$ & 451 & 69.8 & 3.3 & No & DFS + OS \\
\hline 26490984 & Hong et $\mathrm{a}^{20}$ & 2016 & PRC & 7 & 2009-2010 & $55(28-89)$ & 487 & 55 & 1.93 & No & DFS \\
\hline 24532163 & Dirican et $\mathrm{al}^{21}$ & 2015 & Turkey & 6 & 2006-201I & ND & $\mathrm{I}, 527$ & 30 & 4 & Yes & DFS + OS \\
\hline 26214622 & Ulas et $\mathrm{al}^{22}$ & 2015 & Turkey & 7 & 2009-2014 & $51.4 \pm 10.4$ & 187 & 26.0 & 2.38 & No & DFS + OS \\
\hline
\end{tabular}

Notes: 'Koh's study supplied three different cut-off values and the favorite one $(N L R=4)$ was taken in the integrated research. ${ }^{\circ}$ Combined primary and metastasis breast cancer patients. Primary breast cancer patients.

Abbreviations: DFS, disease-free survival; ND, no data; NLR, neutrophil lymphocyte ratio; NOS, Newcastle-Ottawa Quality Assessment Scale; OS, overall survival; PIMD, PubMed unique identifier; PRC, People's Republic of China.

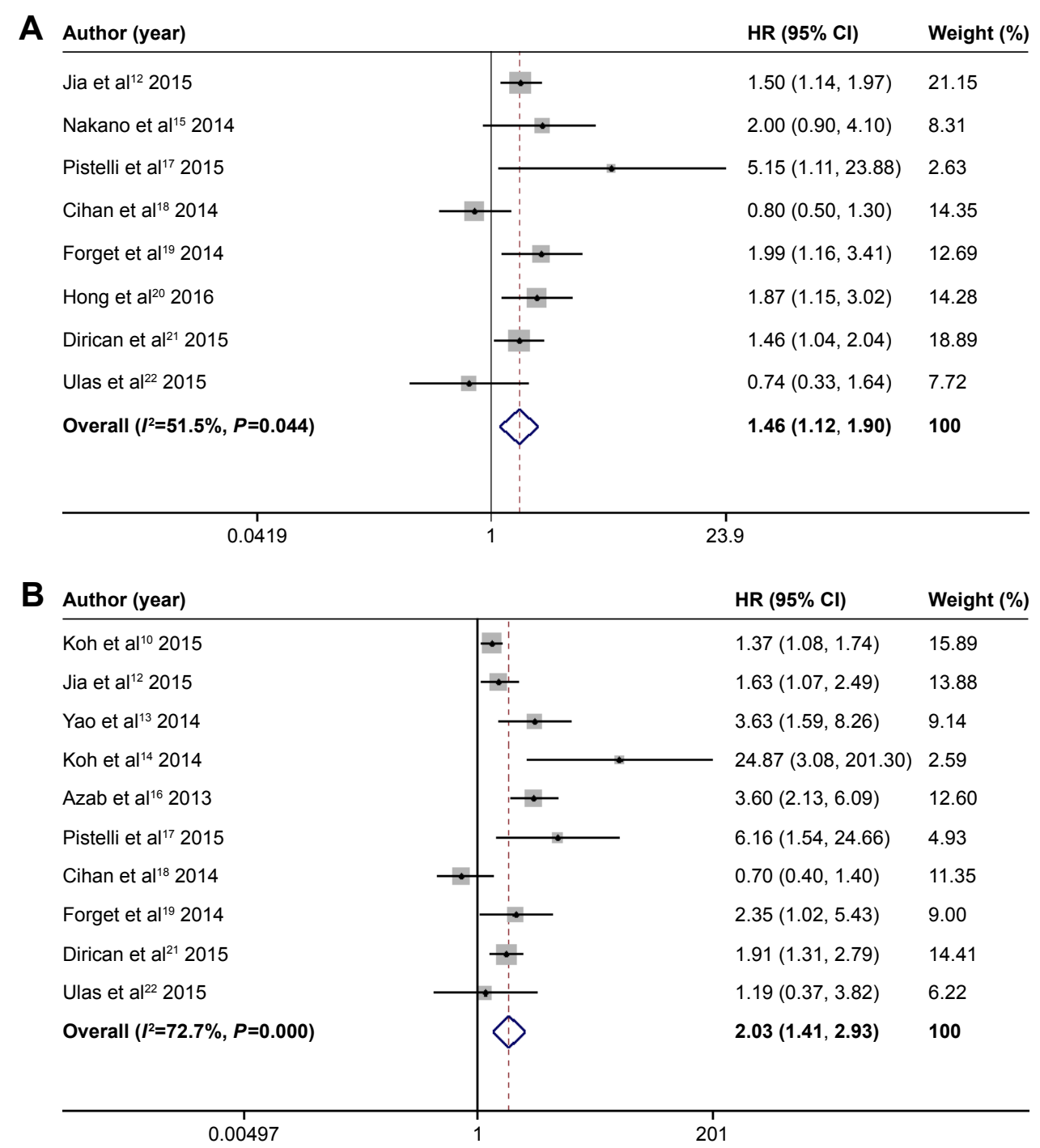

Figure 2 The results of NLR and prognosis of BC in the main analysis.

Notes: (A) Forest plot of HR and 95\% Cl for the association between NLR and DFS in BC patients; (B) Forest plot of HR and $95 \% \mathrm{Cl}$ for the association between PLR and $O S$ in $B C$ patients. Weights are from random effects analysis.

Abbreviations: BC, breast cancer; Cl, confidence interval; DFS, disease-free survival; HR, hazard ratio; $I^{2}$, Higgins I-squared; NLR, neutrophil lymphocyte ratio; OS, overall survival. 

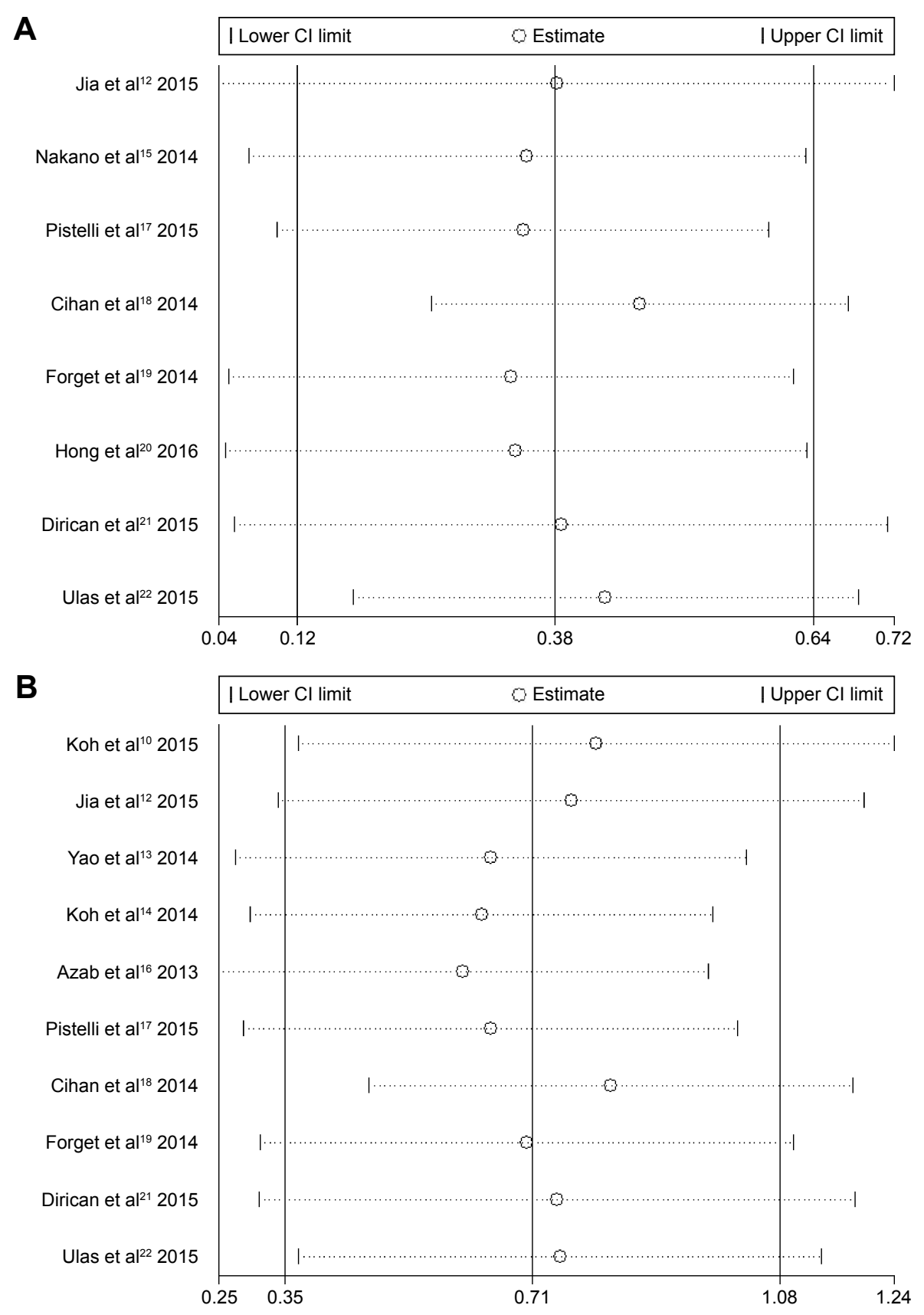

Figure 3 The sensitive analyses of included studies in DFS and OS.

Notes: (A) Included studies in NLR and DFS of BC patients; (B) included studies in NLR and OS of BC patients.

Abbreviations: $\mathrm{BC}$, breast cancer; $\mathrm{Cl}$, confidence interval; DFS, disease-free survival; NLR, neutrophil lymphocyte ratio; OS, overall survival.

and $\mathrm{OS}(P=0.091)$. A Monte Carlo permutation test $(5,000)$ was used for multiple tests. The result failed to reveal the source of the heterogeneity $(P>0.05)$.

\section{NLR, DFS, and OS in univariate analyses}

The data from the univariate analyses in nine studies were extracted and calculated to determine whether the analysis method affected the heterogeneity (Table 5). The pooled results of the univariate analyses were similar to the previous main results.

\section{Publication bias}

Publication bias was examined using Begg's funnel plot and Egger's linear regression test. Publication bias was not obvious in either the DFS or OS studies (DFS: $P=0.386,0.705$; OS: $P=0.283,0.112$ ).

\section{Discussion}

In this meta-analysis, we found that elevated pretreatment NLR was associated with poor prognosis in BC patients. This result was further confirmed by analysis of the HER2+ 
Table 2 Subgroup analyses of NLR and DFS in the breast cancer patients

\begin{tabular}{|c|c|c|c|c|c|}
\hline Subgroup & Detailed & Pooled HR & $95 \% \mathrm{Cl}$ & $I^{2}(\%)$ & $P$-value \\
\hline \multirow[t]{2}{*}{ Region } & Eastern & 1.62 & $1.29-2.03$ & 0 & 0.626 \\
\hline & Western & 1.32 & $0.83-2.08$ & 66.2 & 0.019 \\
\hline \multirow[t]{2}{*}{ Year } & $2013-2014$ & 1.43 & $0.74-2.74$ & 73.7 & 0.022 \\
\hline & $2015-2016$ & 1.51 & $1.15-1.97$ & 36.6 & 0.177 \\
\hline \multirow[t]{2}{*}{ Cut offa } & $<3$ & 1.52 & I.12-2.05 & 30.8 & 0.227 \\
\hline & $\geq 3$ & 1.48 & $0.90-2.45$ & 69.1 & 0.021 \\
\hline \multirow[t]{2}{*}{ Composition } & $M i x^{b}$ & 1.46 & $1.04-2.04$ & - & - \\
\hline & Not $^{c}$ & 1.47 & $1.06-2.05$ & 58.4 & 0.025 \\
\hline \multirow[t]{2}{*}{ Analysis method } & Multivariate & 1.63 & $1.37-1.95$ & 0 & 0.544 \\
\hline & Univariate & 0.78 & $0.52-1.18$ & 0 & 0.87 \\
\hline
\end{tabular}

Notes: ${ }^{\text {TT }}$ The cut-off for classification: the median of value. ${ }^{b}$ Mix: combined primary and metastasis breast cancer. 'Not: primary breast cancer. Eastern region includes China, Japan, Korea, and Malaysia. Western region includes America, Belgium, Italy, and Turkey. Abbreviations: $\mathrm{Cl}$, confidence interval; DFS, disease-free survival; $\mathrm{HR}$, hazard ratio; $I^{2}$, Higgins I-squared; NLR, neutrophil lymphocyte ratio.

and triple-negative breast cancer subtypes. To the best of our knowledge, this study is the largest meta-analysis of the NLR and $\mathrm{BC}$ patients that has ever been performed.

NLR is reported to influence carcinoma diagnosis, classification, and prognosis. NLR is commonly elevated in patients with gastric cancer, lung cancer, colorectal adenocarcinoma, papillary thyroid cancer, and granulosa cell tumors of the ovary. ${ }^{23-27}$ It has also been shown to increase earlier than CEA in patients with stage I/II colorectal cancer. ${ }^{28}$ Furthermore, several studies have reported an association between NLR and tumor grade and stage. A high NLR was observed in patients with G4 glioblastoma compared to those with G3, G2, and G1 glioblastoma. ${ }^{29}$ Pang illustrated that gastric cancer patients with high NLRs had a significant risk of lymph node metastasis. ${ }^{30}$ In the studies of colorectal cancer, researchers from both Turkey and the People's Republic of China found that NLR was closely associated with TNM

Table 3 Subgroup analyses of NLR and OS in the breast cancer patients

\begin{tabular}{|c|c|c|c|c|c|}
\hline Subgroup & Detailed & Pooled HR & $95 \% \mathrm{Cl}$ & $I^{2}(\%)$ & $P$-value \\
\hline \multirow[t]{2}{*}{ Region } & Eastern & 2.17 & $1.22-3.84$ & $74.9 \%$ & 0.008 \\
\hline & Western & 1.97 & I.I3-3.44 & 73.7 & 0.002 \\
\hline \multirow[t]{2}{*}{ Year } & $2013-2014$ & 2.75 & I. $17-6.49$ & 82.5 & $<0.001$ \\
\hline & $2015-2016$ & 1.64 & $1.25-2.14$ & 36.1 & 0.181 \\
\hline \multirow[t]{2}{*}{ Cut off } & $<3$ & 2.61 & $1.14-5.93$ & 67.6 & 0.026 \\
\hline & $\geq 3$ & 1.89 & $1.20-2.97$ & 78.0 & $<0.00$ I \\
\hline \multirow[t]{2}{*}{ Composition } & $M i x^{b}$ & 2.02 & $1.22-3.35$ & $82.4 \%$ & 0.003 \\
\hline & Not $^{c}$ & 2.21 & $1.99-4.11$ & 72.2 & 0.001 \\
\hline \multirow[t]{2}{*}{ Analysis method } & Univariate & 0.79 & $0.45-1.37$ & 0 & 0.432 \\
\hline & Multivariate & 2.41 & $\mid .65-3.5 \mathrm{|}$ & 71.2 & 0.001 \\
\hline
\end{tabular}

Notes: ${ }^{\text {TT }}$ The cut-off for classification: the median of value. ${ }^{b}$ Mix: combined primary and metastasis breast cancer. 'Not: primary breast cancer. Eastern region includes China, Japan, Korea, and Malaysia. Western region includes America, Belgium, Italy, and Turkey. Abbreviations: $\mathrm{Cl}$, confidence interval; HR, hazard ratio; $P^{2}$, Higgins I-squared; NLR, neutrophil lymphocyte ratio; OS, overall survival.
Table 4 NLR and OS in BC subtype patients

\begin{tabular}{|c|c|c|c|c|}
\hline Subtype & Pooled HR & $95 \% \mathrm{Cl}$ & $I^{2}(\%)$ & $P$-value \\
\hline Luminal $\mathrm{A}^{\mathrm{a}}$ & 4.43 & $0.65-30.05$ & 77.7 & 0.011 \\
\hline Luminal $\mathrm{B}^{\mathrm{a}}$ & 1.13 & $0.77-1.67$ & 0 & 0.982 \\
\hline HER2+ & 1.62 & $1.19-2.22$ & 0 & 0.985 \\
\hline TNBC & 2.66 & $1.84-3.86$ & 0 & 0.467 \\
\hline
\end{tabular}

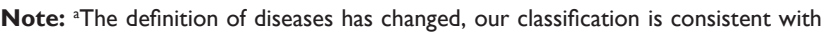
original studies.

Abbreviations: $\mathrm{BC}$, breast cancer; $\mathrm{Cl}$, confidence interval; $\mathrm{HR}$, hazard ratio; HER2, human epidermal growth factor receptor 2; $I^{2}$, Higgins I-squared; NLR, neutrophil lymphocyte ratio; OS, overall survival; TNBC, triple-negative breast cancer.

stage, ${ }^{28,31}$ indicating that NLR might help to identify a reasonable treatment strategy when combined with laboratory and radiographic evaluations. The benefit of treatment of various cancers is difficult to detect; however, a change in NLR may reflect the effectiveness of therapy. The pattern of NLR changes was remarkably different between the responder and nonresponder patients with muscle-invasive bladder cancer receiving neoadjuvant chemotherapy. ${ }^{32}$ The variation between the pre-chemo- and post-chemo-NLR may illustrate the effects of chemotherapy in unresectable or recurrent gastric cancer. ${ }^{33}$ Regarding the prognoses of many malignancies, including respiratory, gastrointestinal, hepatobiliary, gynecological, and urinary cancers, the NLR has been shown to deal with it effectively. The pretreatment NLR in patients with combined small-cell lung cancer was not only strongly associated with the stage of disease and tumor recurrence but could also predict the relapse-free survival and OS as an independent prognostic factor. ${ }^{34}$ This association, however, was not consistent with the studies of esophageal squamous cell cancer. Xie and Hirahara have indicated that NLR failed to have an independent influence on OS, ${ }^{35,36}$ whereas other studies confirmed that the NLR was an independent prognostic factor for both OS and cancer-specific survival. ${ }^{37,38}$ From a multicenter study of gastric cancer, Gunaldi et al found that NLR had a significant prognostic value compared to platelet lymphocyte ratio. ${ }^{39}$ Furthermore, the NLR is better than traditional evaluation systems (Model for End-Stage Liver Disease [MELD] stage and Child-Pugh class) and is used as the prognostic biomarker in patients with

Table 5 Comparison between the results from main analysis and univariate analysis

\begin{tabular}{llllll}
\hline Prognosis & Analysis method & Pooled HR & $\mathbf{9 5 \%} \mathbf{C l}$ & $\boldsymbol{I}^{\boldsymbol{2}}$ (\%) & $\boldsymbol{P}$-value \\
\hline DFS & Main analysis & 1.46 & $\mathrm{I} .12-1.90$ & 51.5 & 0.044 \\
& Univariate analysis & 1.62 & $1.07-2.45$ & 73.8 & 0.002 \\
\multirow{2}{*}{ OS } & Main analysis & 2.03 & $1.41-2.93$ & 72.7 & $<0.00 \mathrm{I}$ \\
& Univariate analysis & 2.79 & $1.49-5.23$ & 83.6 & $<0.00 \mathrm{I}$ \\
\hline
\end{tabular}

Abbreviations: $\mathrm{Cl}$, confidence interval; DFS, disease-free survival; $\mathrm{HR}$, hazardratio; $I^{2}$, Higgins I-squared; OS, overall survival. 
hepatocellular carcinoma. ${ }^{40}$ A prognostic model for survival, which included NLR, was created in a study of patients with advanced pancreatic cancer who were receiving palliative chemotherapy. ${ }^{41}$ When combined with platelet lymphocyte ratio, NLR could be used to stratify patients with endometrial cancer into groups based on risk; the high-risk group had a 2.5-fold higher risk than the low-risk group. ${ }^{42}$ In addition, adrenocortical carcinoma patients with elevated NLRs had a nearly twofold higher risk in an analysis of disease-specific survival and relapse-free survival. ${ }^{43}$

Neutrophil and lymphocyte counts can be altered by various physiological and pathological processes. NLR, however, has been shown to be better than the individual neutrophil, lymphocyte, and total leukocyte counts at predicting the prognosis of BC patients. ${ }^{44}$ At present, the mechanism of the association between NLR and the prognosis of patients with $\mathrm{BC}$ is not completely clear. One study found that neutrophils were stimulated by $\mathrm{BC}$ cancer cells to produce oncostatin $\mathrm{M}$, which affects vascular endothelial growth factor expression and increases $\mathrm{BC}$ cancer cell detachment and invasive capacity. ${ }^{45}$ Furthermore, neutrophils increased the migration of MDA-MB-468 human BC cells through intercellular adhesion molecule- $1 .{ }^{46}$ Recently, Coffelt et al reported that induced by tumor cells, a large number of neutrophils suppressed $\mathrm{CD} 8^{+} \mathrm{T}$ lymphocytes and promoted metastasis through immunosuppression in a single model of BC. ${ }^{47}$ In another study, neutrophils played a key role in metastatic initiation by promoting leukotriene production, which helps the tumor cells to colonize distant tissues. ${ }^{48}$ Neutrophils activated by a2 isoform V-ATPase (a2NTD) had a positive influence on angiogenesis and $\mathrm{BC}$ cell invasiveness. ${ }^{49}$ In addition, it has been known that lymphocytes exhibit antitumor activity in the adaptive immune system. Peripheral lymphocyte count can predict tumor recurrence and survival in both early and metastatic BC. ${ }^{50,51}$ Patients with elevated tumor-infiltrating lymphocyte counts would have better survival, particularly in triple-negative breast cancer and HER2-overexpressing BC. ${ }^{52,53}$ These findings are consistent with our subtype results. These studies show that the NLR may reflect the situations of different inflammatory and immune pathways; in turn, patient prognosis is influenced by both neutrophils and lymphocytes.

In the past 5 years, the predictive role of NLR has been demonstrated in multiple meta-analyses of different types of cancer. Li et al first illustrated that an increased NLR was associated with shorter progression-free survival in a meta-analysis of colorectal cancer. ${ }^{54}$ This association was also noted in metaanalyses of gastric and non-small-cell lung cancer. ${ }^{55,56}$ DFS was worse for patients with elevated NLRs in most of the metaanalyses of gastric cancer, hepatic carcinoma, and esophageal carcinoma. ${ }^{57-59}$ Yodying et al illuminated that there was no association between the NLR and DFS in esophageal carcinoma. ${ }^{60}$ In fact, the associations between NLR and OS in cancer patients have been reported in several meta-analyses. ${ }^{54,55,57,58,61}$

Two meta-analyses on NLR and BC were published in 2014 and 2015..$^{62,63}$ Due to time constraints and improper data processing, there are several limitations in these studies. Firstly, in clinical practice it is important to classify BC into subtypes; however, these were not sufficiently addressed in the studies by Templeton and Chen. ${ }^{62,63}$ The previous meta-analyses also have small sample sizes compared to this study, with 1,195 and 4,293 patients, respectively. ${ }^{62,63}$ We also note that in previous studies by Templeton and Chen, both the studies by Azab were included as independent studies; however, there was some crossover of patients and therefore we have only included the 2013 study in our analysis. The 2012 study by Azab does not have enough data on $\mathrm{HR}$ and $95 \% \mathrm{CI}$. Finally, there has been an increase in publications in this area in the last year with seven new studies on DFS and a further seven new studies on OS, many of which we have included in our analysis. ${ }^{10,12-14,17,20,22}$

\section{Limitations}

Although we carefully calculated the potential association between NLR and prognosis of BC patients from the most recent studies and attempted to derive reliable conclusions for clinical applications, some limitations still exist in our study. First, heterogeneity is a universal problem that may affect the stability of the results from all meta-analyses. Our main results were positive, with high heterogeneity. Based on the results from the subgroup analyses, the analysis method, region, and cut-off value might explain the heterogeneity. Furthermore, the analysis method was nearly considered the source of heterogeneity in the meta-regression of OS $(P<0.01)$. However, when the DFS and OS were calculated using only the data from the univariate analyses, the results failed to achieve the values with homogeneity. Therefore, the heterogeneity might originate from the differences among the studies instead of the analysis method. Second, only five studies were enrolled for the analysis of the association between the NLR and prognosis among the BC patient subtypes. ${ }^{10,12-14,17}$ Therefore, the conclusion is preliminary and should be examined in future studies.

\section{Conclusion}

Overall, this meta-analysis suggests that the pretreatment NLR is associated with the DFS and OS in BC patients. Future studies should perform additional detailed investigations to 
decrease the heterogeneity and determine the appropriate cut-off values for different races.

\section{Acknowledgment}

The authors are grateful to Dr Jennifer Turco (Fiona Stanley Hospital, WA, Australia) for revising the paper.

\section{Disclosure}

The authors report no conflicts of interest in this work.

\section{References}

1. Lozano R, Naghavi M, Foreman K, et al. Global and regional mortality from 235 causes of death for 20 age groups in 1990 and 2010: a systematic analysis for the Global Burden of Disease Study 2010. Lancet. 2012;380(9859):2095-2128.

2. Torre LA, Bray F, Siegel RL, Ferlay J, Lortet-Tieulent J, Jemal A. Global cancer statistics, 2012. CA Cancer J Clin. 2015;65(2):87-108.

3. Jemal A, Bray F, Center MM, Ferlay J, Ward E, Forman D. Global cancer statistics. CA Cancer J Clin. 2011;61(2):69-90.

4. Coates AS, Winer EP, Goldhirsch A, et al. Tailoring therapies-improving the management of early breast cancer: St Gallen International Expert Consensus on the Primary Therapy of Early Breast Cancer 2015. Ann Oncol. 2015;26(8):1533-1546.

5. Coussens LM, Werb Z. Inflammation and cancer. Nature. 2002; 420(6917):860-867

6. Balkwill F, Mantovani A. Inflammation and cancer: back to Virchow? Lancet. 2001;357(9255):539-545.

7. Sicking I, Edlund K, Wesbuer E, et al. Prognostic influence of preoperative C-reactive protein in node-negative breast cancer patients. PLoS One. 2014;9(10):e111306.

8. Cho YA, Sung MK, Yeon JY, Ro J, Kim J. Prognostic role of interleukin-6, interleukin-8, and leptin levels according to breast cancer subtype. Cancer Res Treat. 2013;45(3):210-219.

9. Tripsianis G, Papadopoulou E, Anagnostopoulos K, et al. Coexpression of IL-6 and TNF-alpha: prognostic significance on breast cancer outcome. Neoplasma. 2014;61(2):205-212.

10. Koh $\mathrm{CH}$, Bhoo-Pathy $\mathrm{N}, \mathrm{Ng} \mathrm{KL}$, et al. Utility of pre-treatment neutrophil-lymphocyte ratio and platelet-lymphocyte ratio as prognostic factors in breast cancer. Br J Cancer. 2015;113(1):150-158.

11. Noh H, Eomm M, Han A. Usefulness of pretreatment neutrophil to lymphocyte ratio in predicting disease-specific survival in breast cancer patients. J Breast Cancer. 2013;16(1):55-59.

12. Jia W, Wu J, Jia H, et al. The peripheral blood neutrophil-to-lymphocyte ratio is superior to the lymphocyte-to-monocyte ratio for predicting the long-term survival of triple-negative breast cancer patients. PLoS One. 2015;10(11):e0143061.

13. Yao M, Liu Y, Jin H, et al. Prognostic value of preoperative inflammatory markers in Chinese patients with breast cancer. OncoTargets Ther. 2014; 7:1743-1752.

14. Koh YW, Lee HJ, Ahn JH, Lee JW, Gong G. Prognostic significance of the ratio of absolute neutrophil to lymphocyte counts for breast cancer patients with ER/PR-positivity and HER2-negativity in neoadjuvant setting. Tumour Biol. 2014;35(10):9823-9830.

15. Nakano K, Hosoda M, Yamamoto M, Yamashita H. Prognostic significance of pre-treatment neutrophil: lymphocyte ratio in Japanese patients with breast cancer. Anticancer Res. 2014;34(7):3819-3824.

16. Azab B, Shah N, Radbel J, et al. Pretreatment neutrophil/lymphocyte ratio is superior to platelet/lymphocyte ratio as a predictor of long-term mortality in breast cancer patients. Med Oncol. 2013;30(1):432.

17. Pistelli M, De Lisa M, Ballatore Z, et al. Pre-treatment neutrophil to lymphocyte ratio may be a useful tool in predicting survival in early triple negative breast cancer patients. BMC Cancer. 2015;15:195.
18. Cihan YB, Arslan A, Cetindag MF, Mutlu H. Lack of prognostic value of blood parameters in patients receiving adjuvant radiotherapy for breast cancer. Asian Pac J Cancer Prev. 2014;15(10):4225-4231.

19. Forget P, Bentin C, Machiels JP, Berliere M, Coulie PG, De Kock M. Intraoperative use of ketorolac or diclofenac is associated with improved disease-free survival and overall survival in conservative breast cancer surgery. Br J Anaesth. 2014;113(Suppl 1):i82-i87.

20. Hong J, Mao Y, Chen X, et al. Elevated preoperative neutrophil-tolymphocyte ratio predicts poor disease-free survival in Chinese women with breast cancer. Tumour Biol. 2016;37(3):4135-4142.

21. Dirican A, Kucukzeybek BB, Alacacioglu A, et al. Do the derived neutrophil to lymphocyte ratio and the neutrophil to lymphocyte ratio predict prognosis in breast cancer? Int J Clin Oncol. 2015;20(1):70-81.

22. Ulas A, Avci N, Kos T, et al. Are neutrophil/lymphocyte ratio and platelet/lymphocyte ratio associated with prognosis in patients with HER2-positive early breast cancer receiving adjuvant trastuzumab? J BUON. 2015;20(3):714-722.

23. Lian L, Xia YY, Zhou C, et al. Application of platelet/lymphocyte and neutrophil/lymphocyte ratios in early diagnosis and prognostic prediction in patients with resectable gastric cancer. Cancer Biomark. 2015;15(6):899-907.

24. Kemal Y, Yucel I, Ekiz K, et al. Elevated serum neutrophil to lymphocyte and platelet to lymphocyte ratios could be useful in lung cancer diagnosis. Asian Pac J Cancer Prev. 2014;15(6):2651-2654.

25. Kilincalp S, Coban S, Akinci H, et al. Neutrophil/lymphocyte ratio, platelet/lymphocyte ratio, and mean platelet volume as potential biomarkers for early detection and monitoring of colorectal adenocarcinoma. Eur J Cancer Prev. 2015;24(4):328-333.

26. Kocer D, Karakukcu C, Karaman H, Gokay F, Bayram F. May the neutrophil/lymphocyte ratio be a predictor in the differentiation of different thyroid disorders? Asian Pac J Cancer Prev. 2015;16(9):3875-3879.

27. Yesilyurt H, Tokmak A, Guzel AI, et al. Parameters for predicting granulosa cell tumor of the ovary: a single center retrospective comparative study. Asian Pac J Cancer Prev. 2014;15(19):8447-8450.

28. Jia J, Zheng X, Chen Y, et al. Stage-dependent changes of preoperative neutrophil to lymphocyte ratio and platelet to lymphocyte ratio in colorectal cancer. Tumour Biol. 2015;36(12):9319-9325.

29. Zadora P, Dabrowski W, Czarko K, et al. Preoperative neutrophillymphocyte count ratio helps predict the grade of glial tumor - a pilot study. Neurol Neurochir Pol. 2015;49(1):41-44.

30. Pang W, Lou N, Jin C, et al. Combination of preoperative platelet/ lymphocyte and neutrophil/lymphocyte rates and tumor-related factors to predict lymph node metastasis in patients with gastric cancer. Eur J Gastroenterol Hepatol. 2016;28(5):493-502.

31. Ozgehan G, Kahramanca S, Kaya IO, et al. Neutrophil-lymphocyte ratio as a predictive factor for tumor staging in colorectal cancer. Turk J Med Sci. 2014;44(3):365-368.

32. Seah JA, Leibowitz-Amit R, Atenafu EG, et al. Neutrophil-lymphocyte ratio and pathological response to neoadjuvant chemotherapy in patients with muscle-invasive bladder cancer. Clin Genitourin Cancer. 2015;13(4):e229-e233.

33. Liu H, Song M, Fang F, Gao X, Zhang Z, Wang S. Prediction of chemotherapeutic efficacy using the ratio of neutrophils to lymphocytes in patients with unresectable or recurrent gastric cancer. Oncol Lett. 2015; 10(4):2244-2248.

34. Shao N, Cai Q. High pretreatment neutrophil-lymphocyte ratio predicts recurrence and poor prognosis for combined small cell lung cancer. Clin Transl Oncol. 2015;17(10):772-778.

35. Xie X, Luo KJ, Hu Y, Wang JY, Chen J. Prognostic value of preoperative platelet-lymphocyte and neutrophil-lymphocyte ratio in patients undergoing surgery for esophageal squamous cell cancer. Dis Esophagus. 2016;29(1):79-85.

36. Hirahara N, Matsubara T, Hayashi H, Takai K, Fujii Y, Tajima Y. Impact of inflammation-based prognostic score on survival after curative thoracoscopic esophagectomy for esophageal cancer. Eur J Surg Oncol. 2015;41(10):1308-1315. 
37. Kosumi K, Baba Y, Ishimoto T, et al. Neutrophil/lymphocyte ratio predicts the prognosis in esophageal squamous cell carcinoma patients. Surg Today. 2016;46(4):405-413.

38. Feng JF, Huang Y, Chen QX. The combination of platelet count and neutrophil lymphocyte ratio is a predictive factor in patients with esophageal squamous cell carcinoma. Transl Oncol. 2014;7(5):632-637.

39. Gunaldi M, Goksu S, Erdem D, et al. Prognostic impact of platelet/ lymphocyte and neutrophil/lymphocyte ratios in patients with gastric cancer: a multicenter study. Int J Clin Exp Med. 2015;8(4):5937-5942.

40. Gao F, Li X, Geng M, et al. Pretreatment neutrophil-lymphocyte ratio: an independent predictor of survival in patients with hepatocellular carcinoma. Medicine. 2015;94(11):e639.

41. Kou T, Kanai M, Yamamoto M, et al. Prognostic model for survival based on readily available pretreatment factors in patients with advanced pancreatic cancer receiving palliative chemotherapy. Int J Clin Oncol. 2016;21(1):118-125.

42. Cummings M, Merone L, Keeble C, et al. Preoperative neutrophil: lymphocyte and platelet: lymphocyte ratios predict endometrial cancer survival. Br J Cancer. 2015;113(2):311-320.

43. Bagante F, Tran TB, Postlewait LM, et al. Neutrophil-lymphocyte and platelet-lymphocyte ratio as predictors of disease specific survival after resection of adrenocortical carcinoma. J Surg Oncol. 2015;112(2): 164-172.

44. Azab B, Bhatt VR, Phookan J, et al. Usefulness of the neutrophil-tolymphocyte ratio in predicting short- and long-term mortality in breast cancer patients. Ann Surg Oncol. 2012;19(1):217-224.

45. Queen MM, Ryan RE, Holzer RG, Keller-Peck CR, Jorcyk CL. Breast cancer cells stimulate neutrophils to produce oncostatin $\mathrm{M}$ : potential implications for tumor progression. Cancer Res. 2005;65(19) 8896-8904.

46. Strell C, Lang K, Niggemann B, Zaenker KS, Entschladen F. Neutrophil granulocytes promote the migratory activity of MDA-MB-468 human breast carcinoma cells via ICAM-1. Exp Cell Res. 2010;316(1): $138-148$

47. Coffelt SB, Kersten K, Doornebal CW, et al. IL-17-producing gammadelta $\mathrm{T}$ cells and neutrophils conspire to promote breast cancer metastasis. Nature. 2015;522(7556):345-348.

48. Wculek SK, Malanchi I. Neutrophils support lung colonization of metastasis-initiating breast cancer cells. Nature. 2015;528(7582): 413-417.

49. Ibrahim SA, Katara GK, Kulshrestha A, Jaiswal MK, Amin MA, Beaman KD. Breast cancer associated a2 isoform vacuolar ATPase immunomodulates neutrophils: potential role in tumor progression. Oncotarget. 2015;6(32):33033-33045.
50. De Giorgi U, Mego M, Scarpi E, et al. Relationship between lymphocytopenia and circulating tumor cells as prognostic factors for overall survival in metastatic breast cancer. Clin Breast Cancer. 2012;12(4): 264-269.

51. Pattison CW, Woods KL, Morrison JM. Lymphocytopenia as an independent predictor of early recurrence in breast cancer. Br J Cancer. 1987;55(1):75-76

52. Loi S, Michiels S, Salgado R, et al. Tumor infiltrating lymphocytes are prognostic in triple negative breast cancer and predictive for trastuzumab benefit in early breast cancer: results from the FinHER trial. Ann Oncol. 2014;25(8):1544-1550.

53. Mahmoud SM, Paish EC, Powe DG, et al. Tumor-infiltrating CD8+ lymphocytes predict clinical outcome in breast cancer. J Clin Oncol. 2011;29(15):1949-1955.

54. Li MX, Liu XM, Zhang XF, et al. Prognostic role of neutrophil-tolymphocyte ratio in colorectal cancer: a systematic review and metaanalysis. Int J Cancer. 2014;134(10):2403-2413.

55. Gu XB, Tian T, Tian XJ, Zhang XJ. Prognostic significance of neutrophil-to-lymphocyte ratio in non-small cell lung cancer: a metaanalysis. Sci Rep. 2015;5:12493.

56. Chen J, Hong D, Zhai Y, Shen P. Meta-analysis of associations between neutrophil-to-lymphocyte ratio and prognosis of gastric cancer. World J Surg Oncol. 2015;13:122.

57. Yang X, Huang Y, Feng JF, Liu JS. Prognostic significance of neutrophil-to-lymphocyte ratio in esophageal cancer: a meta-analysis. OncoTargets Ther. 2015;8:789-794.

58. Zhang X, Zhang W, Feng LJ. Prognostic significance of neutrophil lymphocyte ratio in patients with gastric cancer: a meta-analysis. PLoS One. 2014;9(11):e111906.

59. Xue TC, Zhang L, Xie XY, et al. Prognostic significance of the neutrophil-to-lymphocyte ratio in primary liver cancer: a meta-analysis. PLoS One. 2014;9(5):e96072.

60. Yodying H, Matsuda A, Miyashita M, et al. Prognostic significance of neutrophil-to-lymphocyte ratio and platelet-to-lymphocyte ratio in oncologic outcomes of esophageal cancer: a systematic review and meta-analysis. Ann Surg Oncol. 2016;23(2):646-654.

61. Cheng H, Long F, Jaiswar M, Yang L, Wang C, Zhou Z. Prognostic role of the neutrophil-to-lymphocyte ratio in pancreatic cancer: a metaanalysis. Sci Rep. 2015;5:11026.

62. Chen J, Deng Q, Pan Y, et al. Prognostic value of neutrophil-tolymphocyte ratio in breast cancer. FEBS Open Bio. 2015;5:502-507.

63. Templeton AJ, McNamara MG, Seruga B, et al. Prognostic role of neutrophil-to-lymphocyte ratio in solid tumors: a systematic review and meta-analysis. J Natl Cancer Inst. 2014;106(6):dju124.
OncoTargets and Therapy

\section{Publish your work in this journal}

OncoTargets and Therapy is an international, peer-reviewed, open access journal focusing on the pathological basis of all cancers, potential targets for therapy and treatment protocols employed to improve the management of cancer patients. The journal also focuses on the impact of management programs and new therapeutic agents and protocols on

\section{Dovepress}

patient perspectives such as quality of life, adherence and satisfaction. The manuscript management system is completely online and includes a very quick and fair peer-review system, which is all easy to use. Visit http://www.dovepress.com/testimonials.php to read real quotes from published authors. 